Supporting Information for

\title{
Outdoor Residential Water Use Restrictions during Recent Drought Suppressed Disease Vector Abundance in Southern California
}

Abinash Bhattachan ${ }^{1,2, *}$, Nicholas K. Skaff ${ }^{3}$, Amanda M. Irish $^{4}$, Solomon Vimal ${ }^{2}$, Justin V. Remais $^{3}$ and Dennis P. Lettenmaier ${ }^{2}$

${ }^{1}$ Department of Earth and Environmental Sciences, California State University East Bay, Hayward, CA 94542 USA

${ }^{2}$ Department of Geography, University of California, Los Angeles, Los Angeles, CA 90095, USA

${ }^{3}$ Division of Environmental Health Sciences, School of Public Health, University of California, Berkeley, Berkeley, CA 94720, USA

${ }^{4}$ Department of Epidemiology and Biostatistics, School of Medicine, University of California, San Francisco, San Francisco, CA 94158, USA

* corresponding author: abinash.bhattachan@ @ csueastbay.edu

The following document contains supporting information (SI) for "Outdoor residential water use restrictions during recent drought suppressed disease vector abundance in Southern California" by Abinash Bhattachan, Nicholas K. Skaff, Amanda M. Irish, Solomon Vimal, Justin V. Remais, and Dennis P. Lettenmaier. The SI is 16 pages in length and contains the following, 1) details of the random forest model performance (between model predicted $C x$. abundance and surveillance observations) for two study sub-regions for the training period (2006-2013) and counterfactual evaluation period (2014-2016), 2) spatially aggregated (for West LA County and Orange County sub-regions) average weekly (ln) observed and predicted abundance of female Culex quinquefasciatus captured in $\mathrm{CO}_{2}$ traps per trap night from 2010-2016,3) time series of residential daily per capita water use, outdoor residential daily per capita water use, and percent reduction in each month relative to water deliveries in 2013 for each urban water supplier in West LA and Orange County sub-regions, 4) the permutation importance of top 10 environmental predictors for each study sub-region. The two tables and three figures are listed in the table of contents below. 


\section{Table of Contents}

Table S1. Random Forest Model Performance.

Table S2: Average weekly (ln) observed and predicted $C x$. abundance in West LA and Orange County sub-regions in California, 2010-2016. Data for four time periods are: 1) a pre-drought period (2010-2011); 2) the drought period without water restrictions (2012-2013); 3) the drought period with voluntary water use restrictions (2014 and 2016); and 4) the drought period with mandatory water use restrictions (2015).

Figure S1: Time series of average ( \pm standard error) residential daily per capita water use, average ( \pm standard error) outdoor residential daily per capita water use (a, c, e, g) and percent water use reduction in each month relative to the monthly water deliveries in 2013 (b, d, f, g) in urban water suppliers (Hawthorne, Inglewood, Torrance, Golden State Water Company Southwest) in West LA County sub-region.

Figure S2: Time series of average ( \pm standard error) residential daily per capita water use, average ( \pm standard error) outdoor residential daily per capita water use (a, c, e, g, i) and percent water use reduction in each month relative to the monthly water deliveries in 2013 (b, d, f, g, j) in urban water suppliers (Long Beach, Newport Beach, Huntington Beach, Seal Beach, Mesa Water District) in Orange County sub-region.

Figure S3: Permutation variable importance from a) West LA County, b) Orange County subregions showing top 10 environmental predictors. 
Table S1: Random Forest Model Performance

\begin{tabular}{|l|l|l|l|l|l|}
\hline Sub-region & $\begin{array}{l}\text { Training } \\
\text { period } \\
(2006- \\
2013)\end{array}$ & $\begin{array}{l}\text { Counterfactual } \\
\text { evaluation } \\
\text { period }(2014- \\
2016)\end{array}$ & $\mathrm{r}^{2}$ & $\begin{array}{l}\mathrm{r}^{2} \text { Balanced } \\
\text { Annually }\end{array}$ & RMSE \\
\hline West LA County & 1301 & 521 & .31 & .29 & 1.32 \\
\hline Orange County & 2479 & 1172 & .51 & .47 & .86 \\
\hline
\end{tabular}


Table S2: Average weekly (ln) observed and predicted $C x$. abundance in West LA and Orange County sub-regions in California, 2010-2016. Data for four time periods are: 1) a pre-drought period (2010-2011); 2) the drought period without water restrictions (2012-2013); 3) the drought period with voluntary water use restrictions (2014 and 2016); and 4) the drought period with mandatory water use restrictions (2015).

\begin{tabular}{|r|r|l|}
\hline \multicolumn{2}{|l|}{ West LA County } & \\
\hline \multicolumn{1}{|l|}{ date } & \multicolumn{1}{l}{ n_obs } & \multicolumn{1}{l|}{ n_pred } \\
\hline $5 / 31 / 10$ & 1.59524412 & 1.76155521 \\
\hline $6 / 7 / 10$ & 1.58255048 & 2.02705447 \\
\hline $6 / 14 / 10$ & 1.0479199 & 1.66922009 \\
\hline $6 / 21 / 10$ & 2.01468976 & 1.83254043 \\
\hline $6 / 28 / 10$ & 1.87917454 & 1.67848324 \\
\hline $7 / 5 / 10$ & 2.27909214 & 1.75439333 \\
\hline $7 / 12 / 10$ & 1.79111487 & 2.04942281 \\
\hline $7 / 19 / 10$ & 2.24524169 & 2.35217662 \\
\hline $7 / 26 / 10$ & 2.327839 & 1.85719932 \\
\hline $8 / 2 / 10$ & 1.6956428 & 1.60778685 \\
\hline $8 / 9 / 10$ & 1.88526457 & 1.75672701 \\
\hline $8 / 16 / 10$ & 2.13894061 & 1.89405591 \\
\hline $8 / 23 / 10$ & 2.38433653 & 2.20175572 \\
\hline $8 / 30 / 10$ & 1.53984074 & 1.47885015 \\
\hline $9 / 6 / 10$ & 1.55380486 & 1.71842402 \\
\hline $9 / 13 / 10$ & 1.7570702 & 1.66010941 \\
\hline $9 / 20 / 10$ & 1.79168588 & 1.62995851 \\
\hline $9 / 27 / 10$ & 1.80982913 & 2.13369512 \\
\hline $10 / 4 / 10$ & 0.90396242 & 1.51685921 \\
\hline $10 / 11 / 10$ & 1.28140113 & 1.74812838 \\
\hline $10 / 18 / 10$ & 0.65298413 & 1.27733677 \\
\hline $10 / 25 / 10$ & 0.8914632 & 1.30188745 \\
\hline $11 / 1 / 10$ & 1.25831383 & 1.46531015 \\
\hline $11 / 8 / 10$ & 0.64811796 & 1.24183404 \\
\hline $11 / 15 / 10$ & 0.74661629 & 1.12602153 \\
\hline $11 / 22 / 10$ & 0.55302872 & 0.66335729 \\
\hline $11 / 29 / 10$ & 0.09902103 & 0.6428875 \\
\hline $5 / 30 / 11$ & 1.02474472 & 1.4099128 \\
\hline & & \\
\hline
\end{tabular}




\begin{tabular}{|r|r|r|}
\hline $6 / 6 / 11$ & 2.14920345 & 1.76200323 \\
\hline $6 / 13 / 11$ & 1.27603456 & 1.32704906 \\
\hline $6 / 20 / 11$ & 2.10638664 & 1.87592402 \\
\hline $6 / 27 / 11$ & 2.55551006 & 2.12045413 \\
\hline $7 / 4 / 11$ & 1.06262622 & 2.1150954 \\
\hline $7 / 11 / 11$ & 2.92000155 & 2.18178868 \\
\hline $7 / 18 / 11$ & 1.77632725 & 2.00272176 \\
\hline $7 / 25 / 11$ & 2.61153675 & 2.48999767 \\
\hline $8 / 1 / 11$ & 2.0592106 & 2.19194318 \\
\hline $8 / 8 / 11$ & 2.33356308 & 2.13409898 \\
\hline $8 / 15 / 11$ & 1.86218877 & 1.74068168 \\
\hline $8 / 22 / 11$ & 2.1976054 & 1.96993112 \\
\hline $8 / 29 / 11$ & 2.26880793 & 1.9372416 \\
\hline $9 / 5 / 11$ & 2.21718061 & 2.05923136 \\
\hline $9 / 12 / 11$ & 1.91397452 & 1.64409797 \\
\hline $9 / 19 / 11$ & 2.07856157 & 1.88402282 \\
\hline $9 / 26 / 11$ & 1.88011646 & 1.96687044 \\
\hline $10 / 3 / 11$ & 1.00703641 & 1.33733379 \\
\hline $10 / 10 / 11$ & 1.55212271 & 1.67722928 \\
\hline $10 / 17 / 11$ & 1.19908973 & 1.37022583 \\
\hline $10 / 24 / 11$ & 1.87136015 & 1.29004043 \\
\hline $10 / 31 / 11$ & 1.42313263 & 1.36485405 \\
\hline $11 / 7 / 11$ & 0.78076335 & 1.17793411 \\
\hline $11 / 14 / 11$ & 0.86697839 & 0.88965528 \\
\hline $11 / 21 / 11$ & 0.13862944 & 0.77129845 \\
\hline $11 / 28 / 11$ & 0.41588831 & 1.48765869 \\
\hline $5 / 28 / 12$ & & 0.78063115 \\
\hline $6 / 4 / 12$ & 2.25082735 & 1.60787709 \\
\hline $6 / 11 / 12$ & 1.61527624 & 1.67691697 \\
\hline $6 / 18 / 12$ & 1.84857626 & 1.67004392 \\
\hline $6 / 25 / 12$ & 2.46383231 & 2.08601878 \\
\hline $7 / 2 / 12$ & 2.08774375 & 1.52726305 \\
\hline $7 / 9 / 12$ & 2.71275348 & 2.32685475 \\
\hline $7 / 16 / 12$ & 2.64158467 & 2.13448514 \\
\hline $7 / 23 / 12$ & 2.30830839 & 1.99505308 \\
\hline $7 / 30 / 12$ & 2.42559654 & 1.88574232 \\
\hline $8 / 6 / 12$ & 2.92968057 & 2.52835771 \\
\hline $8 / 13 / 12$ & 2.32241313 & 2.15628147 \\
\hline $8 / 20 / 12$ & 1.94065503 & 2.34445082 \\
\hline & & \\
\hline
\end{tabular}




\begin{tabular}{|r|r|r|}
\hline $8 / 27 / 12$ & 1.67127787 & 2.45077957 \\
\hline $9 / 3 / 12$ & 1.65391747 & 2.05131708 \\
\hline $9 / 10 / 12$ & 2.1468832 & 2.30415598 \\
\hline $9 / 17 / 12$ & 1.80077614 & 2.1538854 \\
\hline $9 / 24 / 12$ & 2.12066619 & 2.08374635 \\
\hline $10 / 1 / 12$ & 1.75925419 & 2.17351827 \\
\hline $10 / 8 / 12$ & 2.08162347 & 1.80998477 \\
\hline $10 / 15 / 12$ & 1.86237445 & 2.11619237 \\
\hline $10 / 22 / 12$ & 1.71973343 & 1.52220195 \\
\hline $10 / 29 / 12$ & 1.65681647 & 1.79745842 \\
\hline $11 / 5 / 12$ & 2.11567455 & 1.99623449 \\
\hline $11 / 12 / 12$ & 1.84784432 & 1.51105368 \\
\hline $11 / 26 / 12$ & 1.24847859 & 1.22983662 \\
\hline $6 / 3 / 13$ & 1.49926873 & 1.87333137 \\
\hline $6 / 10 / 13$ & 1.46325898 & 2.32457511 \\
\hline $6 / 17 / 13$ & 2.20642775 & 2.17664848 \\
\hline $6 / 24 / 13$ & 1.81332195 & 2.25595279 \\
\hline $7 / 1 / 13$ & 2.1095375 & 2.3347662 \\
\hline $7 / 8 / 13$ & 1.81976096 & 2.38223996 \\
\hline $7 / 15 / 13$ & 1.37231606 & 1.93508816 \\
\hline $7 / 22 / 13$ & 2.28990527 & 2.1863278 \\
\hline $7 / 29 / 13$ & 1.89927986 & 1.87992709 \\
\hline $8 / 5 / 13$ & 1.60696634 & 1.69837547 \\
\hline $8 / 12 / 13$ & 1.68584317 & 2.12892875 \\
\hline $8 / 19 / 13$ & 1.48896079 & 1.9974549 \\
\hline $8 / 26 / 13$ & 2.12849573 & 2.37604297 \\
\hline $9 / 2 / 13$ & 1.47075201 & 2.10727559 \\
\hline $9 / 9 / 13$ & 1.78507338 & 1.90031539 \\
\hline $9 / 16 / 13$ & 1.32217757 & 1.72886651 \\
\hline $9 / 23 / 13$ & 1.55847767 & 1.99095299 \\
\hline $9 / 30 / 13$ & 0.91373601 & 1.63923333 \\
\hline $10 / 7 / 13$ & 1.45059689 & 1.93067285 \\
\hline $10 / 14 / 13$ & 1.38638136 & 1.65098779 \\
\hline $10 / 21 / 13$ & 1.40256029 & 1.62556955 \\
\hline $10 / 28 / 13$ & 0.8862596 & 1.4633903 \\
\hline $11 / 4 / 13$ & 1.02728079 & 1.33040999 \\
\hline $11 / 11 / 13$ & 1.21086803 & 1.67113415 \\
\hline $11 / 18 / 13$ & 0.79216589 & 1.27169486 \\
\hline $11 / 25 / 13$ & 0.1732868 & 0.90325833 \\
\hline & & \\
\hline
\end{tabular}




\begin{tabular}{|r|r|r|}
\hline $6 / 2 / 14$ & 1.72693329 & 1.81722211 \\
\hline $6 / 9 / 14$ & 2.21014917 & 2.06281701 \\
\hline $6 / 16 / 14$ & 0.75503184 & 1.27609032 \\
\hline $6 / 23 / 14$ & 2.11021303 & 2.17147664 \\
\hline $6 / 30 / 14$ & 1.1370329 & 1.65601442 \\
\hline $7 / 7 / 14$ & 1.88270824 & 2.10784797 \\
\hline $7 / 14 / 14$ & 2.07316234 & 1.72263947 \\
\hline $7 / 21 / 14$ & 2.46165061 & 2.07297595 \\
\hline $7 / 28 / 14$ & 2.34719296 & 2.24459311 \\
\hline $8 / 4 / 14$ & 2.4411314 & 1.76230919 \\
\hline $8 / 11 / 14$ & 2.46877202 & 2.03958952 \\
\hline $8 / 18 / 14$ & 1.80570971 & 1.82281135 \\
\hline $8 / 25 / 14$ & 2.80489712 & 2.1175518 \\
\hline $9 / 1 / 14$ & 1.36202307 & 1.62408685 \\
\hline $9 / 8 / 14$ & 1.85395862 & 1.79460048 \\
\hline $9 / 15 / 14$ & 2.06266351 & 2.12183621 \\
\hline $9 / 22 / 14$ & 2.04190546 & 1.72453621 \\
\hline $9 / 29 / 14$ & 1.4405931 & 1.75803033 \\
\hline $10 / 6 / 14$ & 1.76294299 & 2.03361466 \\
\hline $10 / 13 / 14$ & 2.49677684 & 1.85761341 \\
\hline $10 / 20 / 14$ & 1.81573712 & 1.9066179 \\
\hline $10 / 27 / 14$ & 1.89918164 & 1.88559096 \\
\hline $11 / 3 / 14$ & 1.63202795 & 1.59309626 \\
\hline $11 / 10 / 14$ & 0.93638595 & 1.67704479 \\
\hline $11 / 17 / 14$ & 1.64478413 & 1.26301339 \\
\hline $11 / 24 / 14$ & 0.27465307 & 0.89335033 \\
\hline $5 / 30 / 16$ & 1.11829685 & 1.25195262 \\
\hline $6 / 6 / 16$ & 2.47116737 & 2.14180811 \\
\hline $6 / 13 / 16$ & 1.82185049 & 1.65031275 \\
\hline $6 / 20 / 16$ & 2.01969531 & 2.58782104 \\
\hline $6 / 27 / 16$ & 1.92515075 & 2.26314193 \\
\hline $7 / 4 / 16$ & 2.30893336 & 2.64531341 \\
\hline $7 / 11 / 16$ & 1.96725842 & 2.20536712 \\
\hline $7 / 18 / 16$ & 2.24992541 & 2.55864956 \\
\hline $7 / 25 / 16$ & 1.62350923 & 2.18788639 \\
\hline $8 / 1 / 16$ & 1.82347982 & 2.48245805 \\
\hline $8 / 8 / 16$ & 1.68221844 & 2.06353048 \\
\hline $8 / 15 / 16$ & 1.47959332 & 2.57043128 \\
\hline $8 / 22 / 16$ & 1.20485723 & 2.15061889 \\
\hline & & \\
\hline
\end{tabular}




\begin{tabular}{|r|r|r|}
\hline $8 / 29 / 16$ & 1.84640852 & 2.69816974 \\
\hline $9 / 5 / 16$ & 1.35246934 & 1.91586199 \\
\hline $9 / 12 / 16$ & 2.22503469 & 2.03714532 \\
\hline $9 / 19 / 16$ & 1.5209512 & 2.07093119 \\
\hline $9 / 26 / 16$ & 1.68635468 & 2.57163927 \\
\hline $10 / 3 / 16$ & 1.7307631 & 1.64040786 \\
\hline $10 / 10 / 16$ & 1.88263453 & 1.99664815 \\
\hline $10 / 17 / 16$ & 0.9806295 & 1.64030679 \\
\hline $10 / 24 / 16$ & 1.66846805 & 1.91779173 \\
\hline $10 / 31 / 16$ & 0.93203512 & 1.27713884 \\
\hline $11 / 7 / 16$ & 2.47993863 & 2.19153846 \\
\hline $11 / 14 / 16$ & 1.88215035 & 1.903502 \\
\hline $11 / 21 / 16$ & 0.80507034 & 1.03276319 \\
\hline $11 / 28 / 16$ & 1.09861229 & 0.92001436 \\
\hline $6 / 1 / 15$ & 0.82338084 & 1.47414063 \\
\hline $6 / 8 / 15$ & 0.91760671 & 1.79900656 \\
\hline $6 / 15 / 15$ & 1.79840867 & 1.74277922 \\
\hline $6 / 22 / 15$ & 2.26567242 & 2.37362841 \\
\hline $6 / 29 / 15$ & 1.29709458 & 2.06931464 \\
\hline $7 / 6 / 15$ & 2.56939958 & 2.29860362 \\
\hline $7 / 13 / 15$ & 2.0371292 & 1.97742208 \\
\hline $7 / 20 / 15$ & 2.46030142 & 2.21649543 \\
\hline $7 / 27 / 15$ & 1.6690801 & 2.23823579 \\
\hline $8 / 3 / 15$ & 1.53662251 & 1.98403143 \\
\hline $8 / 10 / 15$ & 1.59903225 & 2.27736879 \\
\hline $8 / 17 / 15$ & 1.26117561 & 1.83583979 \\
\hline $8 / 24 / 15$ & 1.26995503 & 2.25237495 \\
\hline $8 / 31 / 15$ & 1.1555505 & 1.79627064 \\
\hline $9 / 7 / 15$ & 1.02358614 & 2.20090593 \\
\hline $9 / 14 / 15$ & 1.55764448 & 1.82669064 \\
\hline $9 / 21 / 15$ & 1.5738148 & 2.17350082 \\
\hline $9 / 28 / 15$ & 1.75963785 & 1.90037981 \\
\hline $10 / 5 / 15$ & 1.27644504 & 1.8955047 \\
\hline $10 / 12 / 15$ & 1.66664065 & 1.72063016 \\
\hline $10 / 19 / 15$ & 1.42832287 & 1.71477276 \\
\hline $10 / 26 / 15$ & 1.16833031 & 1.65036422 \\
\hline $11 / 2 / 15$ & 2.09196897 & 1.63508479 \\
\hline $11 / 9 / 15$ & 0.54161004 & 1.4521468 \\
\hline $11 / 16 / 15$ & 0.74655702 & 1.18729072 \\
\hline & & \\
\hline
\end{tabular}




\begin{tabular}{|c|c|c|}
\hline $11 / 23 / 15$ & 1.29040034 & 1.39805168 \\
\hline \multicolumn{3}{|c|}{ Orange County } \\
\hline date & ln_obs & ln_pred \\
\hline $5 / 31 / 10$ & 1.97374474 & 1.79514974 \\
\hline $6 / 7 / 10$ & 2.08326984 & 1.63550833 \\
\hline $6 / 14 / 10$ & 1.70178099 & 1.61498624 \\
\hline $6 / 21 / 10$ & 2.06740327 & 1.63652221 \\
\hline $6 / 28 / 10$ & 0.94578647 & 1.27602059 \\
\hline $7 / 5 / 10$ & 1.20691935 & 1.18560258 \\
\hline $7 / 12 / 10$ & 2.24133424 & 2.13563108 \\
\hline 7/19/10 & 1.81388686 & 1.61968626 \\
\hline $7 / 26 / 10$ & 1.91264128 & 1.62440474 \\
\hline $8 / 2 / 10$ & 1.77237537 & 1.39194395 \\
\hline $8 / 9 / 10$ & 1.9980199 & 1.57416595 \\
\hline $8 / 16 / 10$ & 2.3479771 & 1.53245779 \\
\hline $8 / 23 / 10$ & 1.5684676 & 1.41388203 \\
\hline $8 / 30 / 10$ & 1.35702979 & 1.15699393 \\
\hline $9 / 6 / 10$ & 1.0092524 & 1.17357029 \\
\hline $9 / 13 / 10$ & 1.07888661 & 1.09254696 \\
\hline $9 / 20 / 10$ & 1.06516887 & 1.0368307 \\
\hline $9 / 27 / 10$ & 1.21847982 & 1.22211307 \\
\hline $10 / 4 / 10$ & 0.23104906 & 0.73424105 \\
\hline $10 / 11 / 10$ & 0.84138922 & 0.92973193 \\
\hline $10 / 18 / 10$ & 0.1732868 & 0.66597549 \\
\hline $10 / 25 / 10$ & 0.89817338 & 1.31382217 \\
\hline $11 / 1 / 10$ & 1.53924253 & 1.38722501 \\
\hline $11 / 8 / 10$ & 1.09861229 & 1.5921104 \\
\hline $11 / 15 / 10$ & 1.58902692 & 1.59631228 \\
\hline $5 / 30 / 11$ & 1.86017856 & 1.94793952 \\
\hline $6 / 6 / 11$ & 0.58045634 & 0.82518274 \\
\hline $6 / 13 / 11$ & 0.75728192 & 1.14361128 \\
\hline $6 / 20 / 11$ & 0.90853219 & 1.34286433 \\
\hline $6 / 27 / 11$ & 1.31955018 & 1.61648728 \\
\hline $7 / 4 / 11$ & 0.92055818 & 1.31298106 \\
\hline $7 / 11 / 11$ & 1.16446092 & 1.39299987 \\
\hline $7 / 18 / 11$ & 1.26537127 & 1.35609262 \\
\hline $7 / 25 / 11$ & 1.23771177 & 1.34742294 \\
\hline $8 / 1 / 11$ & 1.31257603 & 1.4920598 \\
\hline $8 / 8 / 11$ & 0.63765187 & 0.98958099 \\
\hline
\end{tabular}




\begin{tabular}{|r|r|r|}
\hline $8 / 15 / 11$ & 0.52967564 & 0.89946727 \\
\hline $8 / 22 / 11$ & 0.83765547 & 1.18117902 \\
\hline $8 / 29 / 11$ & 0.83024612 & 1.11280567 \\
\hline $9 / 5 / 11$ & 1.11478008 & 1.15717646 \\
\hline $9 / 12 / 11$ & 0.74406345 & 0.91286732 \\
\hline $9 / 19 / 11$ & 0.74792451 & 0.97052591 \\
\hline $9 / 26 / 11$ & 0.76543644 & 0.91785117 \\
\hline $10 / 3 / 11$ & 0.82830222 & 1.35567033 \\
\hline $10 / 10 / 11$ & 0.78721576 & 0.86890179 \\
\hline $10 / 17 / 11$ & 0.50252394 & 0.74320062 \\
\hline $10 / 24 / 11$ & 0.65616419 & 0.53275411 \\
\hline $10 / 31 / 11$ & 1.08619803 & 0.77272758 \\
\hline $11 / 7 / 11$ & 0.54461564 & 0.65188673 \\
\hline $11 / 14 / 11$ & 0.15694461 & 0.61325782 \\
\hline $11 / 21 / 11$ & 0.1732868 & 0.48335546 \\
\hline $11 / 28 / 11$ & 0.32188758 & 0.54786056 \\
\hline $5 / 28 / 12$ & & 0.46274123 \\
\hline $6 / 4 / 12$ & 1.21617563 & 1.19190921 \\
\hline $6 / 11 / 12$ & 1.17342565 & 1.28231073 \\
\hline $6 / 18 / 12$ & 0.79686656 & 1.13227281 \\
\hline $6 / 25 / 12$ & 1.2058596 & 1.42353426 \\
\hline $7 / 2 / 12$ & 0.845765 & 1.25330483 \\
\hline $7 / 9 / 12$ & 0.99310151 & 1.26675982 \\
\hline $7 / 16 / 12$ & 1.25453773 & 1.30737274 \\
\hline $7 / 23 / 12$ & 0.90880391 & 1.10273606 \\
\hline $7 / 30 / 12$ & 0.88555726 & 1.10338487 \\
\hline $8 / 6 / 12$ & 1.3289779 & 1.34061222 \\
\hline $8 / 13 / 12$ & 1.14136702 & 1.36292662 \\
\hline $8 / 20 / 12$ & 0.77003811 & 1.3764734 \\
\hline $8 / 27 / 12$ & 0.75143035 & 1.11407548 \\
\hline $9 / 3 / 12$ & 0.28891399 & 0.65754508 \\
\hline $9 / 10 / 12$ & 1.00512627 & 1.1719171 \\
\hline $9 / 17 / 12$ & 0.54827093 & 0.91499714 \\
\hline $9 / 24 / 12$ & 0.52318817 & 1.04940286 \\
\hline $10 / 1 / 12$ & 0.90589605 & 1.31045067 \\
\hline $10 / 8 / 12$ & 0.41865184 & 0.87698666 \\
\hline $10 / 15 / 12$ & 0.87536252 & 1.37698045 \\
\hline $10 / 22 / 12$ & 0.73204731 & 1.06990668 \\
\hline $10 / 29 / 12$ & 0.15890269 & 0.64581933 \\
\hline & & \\
\hline
\end{tabular}




\begin{tabular}{|r|r|r|}
\hline $11 / 5 / 12$ & 0.17917595 & 0.52756546 \\
\hline $6 / 3 / 13$ & 0.92527833 & 1.12846667 \\
\hline $6 / 10 / 13$ & 1.43209552 & 1.51517617 \\
\hline $6 / 17 / 13$ & 1.40816208 & 1.37937938 \\
\hline $6 / 24 / 13$ & 1.48181215 & 1.22709878 \\
\hline $7 / 1 / 13$ & 0.28881133 & 0.59200809 \\
\hline $7 / 8 / 13$ & 0.90202906 & 1.25017538 \\
\hline $7 / 15 / 13$ & 0.7699199 & 1.05455366 \\
\hline $7 / 22 / 13$ & 1.10351732 & 1.24674357 \\
\hline $7 / 29 / 13$ & 0.69835018 & 1.07710655 \\
\hline $8 / 5 / 13$ & 0.97829664 & 1.21283769 \\
\hline $8 / 12 / 13$ & 0.79332763 & 0.986213 \\
\hline $8 / 19 / 13$ & 1.05513971 & 1.24245589 \\
\hline $8 / 26 / 13$ & 0.8794419 & 0.9610254 \\
\hline $9 / 2 / 13$ & 0.48860139 & 0.8160707 \\
\hline $9 / 9 / 13$ & 1.35208129 & 1.2268991 \\
\hline $9 / 16 / 13$ & 0.71173209 & 0.98072502 \\
\hline $9 / 23 / 13$ & 0.78621843 & 0.98473713 \\
\hline $9 / 30 / 13$ & 0.63238384 & 0.81212109 \\
\hline $10 / 7 / 13$ & 0.37092549 & 0.95216651 \\
\hline $10 / 14 / 13$ & 0.63109986 & 0.871581 \\
\hline $10 / 21 / 13$ & 0.90204321 & 0.86048775 \\
\hline $10 / 28 / 13$ & 0.4537783 & 0.6681245 \\
\hline $11 / 4 / 13$ & 0.36398545 & 0.754384 \\
\hline $11 / 11 / 13$ & 0.53629661 & 0.88152152 \\
\hline $11 / 18 / 13$ & 1.47152601 & 1.87489373 \\
\hline $6 / 2 / 14$ & 0.5908816 & 0.97808329 \\
\hline $6 / 9 / 14$ & 1.22739217 & 1.29033259 \\
\hline $6 / 16 / 14$ & 1.1488873 & 1.38109774 \\
\hline $6 / 23 / 14$ & 0.49742168 & 0.73645136 \\
\hline $6 / 30 / 14$ & 0.58700009 & 1.02125577 \\
\hline $7 / 7 / 14$ & 1.40806535 & 1.6109161 \\
\hline $7 / 14 / 14$ & 0.8410043 & 1.389773 \\
\hline $7 / 21 / 14$ & 1.24424823 & 1.37676505 \\
\hline $7 / 28 / 14$ & 0.78766257 & 1.26616633 \\
\hline $8 / 4 / 14$ & 1.04134132 & 1.32856218 \\
\hline $8 / 11 / 14$ & 1.04947324 & 1.174917 \\
\hline $8 / 18 / 14$ & 0.58416615 & 1.33219202 \\
\hline $8 / 25 / 14$ & 0.75815862 & 1.1901704 \\
\hline & & \\
\hline
\end{tabular}




\begin{tabular}{|c|c|c|}
\hline $9 / 1 / 14$ & 1.0128439 & 1.32162238 \\
\hline $9 / 8 / 14$ & 1.27093229 & 0.89150273 \\
\hline $9 / 15 / 14$ & 1.10385642 & 1.13761087 \\
\hline $9 / 22 / 14$ & 0.57807435 & 1.17688517 \\
\hline $9 / 29 / 14$ & 0.7648069 & 1.11381126 \\
\hline $10 / 6 / 14$ & 0.79769674 & 0.97191289 \\
\hline $10 / 13 / 14$ & 0.91286964 & 1.05403023 \\
\hline $10 / 20 / 14$ & 1.2688875 & 1.60818103 \\
\hline $10 / 27 / 14$ & 0.6025184 & 0.86941399 \\
\hline $11 / 3 / 14$ & 0.16288722 & 0.64463221 \\
\hline $11 / 10 / 14$ & 0.10986123 & 0.58098188 \\
\hline $11 / 17 / 14$ & 0.45429203 & 0.6249432 \\
\hline $11 / 24 / 14$ & 0 & 0.56562192 \\
\hline $5 / 30 / 16$ & 0.57472732 & 0.74047523 \\
\hline $6 / 6 / 16$ & 0.60853481 & 0.80565478 \\
\hline $6 / 13 / 16$ & 0.87526604 & 0.90717602 \\
\hline $6 / 20 / 16$ & 0.54391055 & 0.87489473 \\
\hline $6 / 27 / 16$ & 0.82504541 & 1.12510451 \\
\hline $7 / 4 / 16$ & 0.94860184 & 0.70749212 \\
\hline $7 / 11 / 16$ & 0.82561928 & 0.85564358 \\
\hline $7 / 18 / 16$ & 0.71674396 & 1.01468126 \\
\hline $7 / 25 / 16$ & 0.57188462 & 0.71909598 \\
\hline $8 / 1 / 16$ & 0.58377956 & 0.90491913 \\
\hline $8 / 8 / 16$ & 0.27803833 & 0.71161355 \\
\hline $8 / 15 / 16$ & 0.68583717 & 0.85479347 \\
\hline $8 / 22 / 16$ & 0.71727234 & 1.04578425 \\
\hline $8 / 29 / 16$ & 0.60909657 & 0.92042336 \\
\hline $9 / 5 / 16$ & 0.47539777 & 0.68626426 \\
\hline $9 / 12 / 16$ & 0.41801809 & 0.68400235 \\
\hline 9/19/16 & 0.54761083 & 1.00929822 \\
\hline $9 / 26 / 16$ & 0.95163447 & 1.09463508 \\
\hline $10 / 3 / 16$ & 0.28197019 & 0.57599172 \\
\hline $10 / 10 / 16$ & 0.65621298 & 0.77650701 \\
\hline $10 / 17 / 16$ & 1.08299863 & 0.8535554 \\
\hline $10 / 24 / 16$ & 0.41282864 & 0.69847319 \\
\hline $10 / 31 / 16$ & 0.6877004 & 0.59973944 \\
\hline $11 / 7 / 16$ & 0.47670808 & 0.58481446 \\
\hline $11 / 14 / 16$ & 0.52918122 & 0.96013391 \\
\hline $11 / 21 / 16$ & 0.59725316 & 0.71493231 \\
\hline
\end{tabular}




\begin{tabular}{|r|r|r|}
\hline $11 / 28 / 16$ & 0.11552453 & 0.52138268 \\
\hline $6 / 1 / 15$ & 0.5817262 & 1.13567828 \\
\hline $6 / 8 / 15$ & 0.33132089 & 1.13482658 \\
\hline $6 / 15 / 15$ & 0.46847137 & 0.99897597 \\
\hline $6 / 22 / 15$ & 0.38632955 & 0.90072862 \\
\hline $6 / 29 / 15$ & 0.69970713 & 0.96085001 \\
\hline $7 / 6 / 15$ & 0.91561888 & 0.81774334 \\
\hline $7 / 13 / 15$ & 0.90441099 & 0.9670499 \\
\hline $7 / 20 / 15$ & 0.78312602 & 0.9651644 \\
\hline $7 / 27 / 15$ & 0.61915343 & 1.04447047 \\
\hline $8 / 3 / 15$ & 1.20705594 & 1.32254466 \\
\hline $8 / 10 / 15$ & 0.65759703 & 1.04930456 \\
\hline $8 / 17 / 15$ & 1.27589844 & 1.8675932 \\
\hline $8 / 24 / 15$ & 0.5241747 & 0.86696693 \\
\hline $8 / 31 / 15$ & 0.30718702 & 0.80897338 \\
\hline $9 / 7 / 15$ & 0.56362005 & 0.95018143 \\
\hline $9 / 14 / 15$ & 0.26147102 & 0.8624012 \\
\hline $9 / 21 / 15$ & 0.15342246 & 0.89472274 \\
\hline $9 / 28 / 15$ & 0.61360048 & 1.09794258 \\
\hline $10 / 5 / 15$ & 0.811528 & 0.99820926 \\
\hline $10 / 12 / 15$ & 0.6387602 & 1.01618554 \\
\hline $10 / 19 / 15$ & 0.63252045 & 1.00443173 \\
\hline $10 / 26 / 15$ & 0.51499978 & 0.83004594 \\
\hline $11 / 2 / 15$ & 0.58815063 & 0.85394695 \\
\hline $11 / 9 / 15$ & 0.35695748 & 0.7411217 \\
\hline $11 / 16 / 15$ & 0.32602487 & 0.78528477 \\
\hline $11 / 23 / 15$ & 0.1732868 & 0.71435501 \\
\hline & & \\
\hline
\end{tabular}


Figure S1: Time series of average ( \pm standard error) residential daily per capita water use, average ( \pm standard error) outdoor residential daily per capita water use (a, c, e, g) and percent water use reduction in each month relative to the monthly water deliveries in 2013 (b, d, f, g) in urban water suppliers (Hawthorne, Inglewood, Torrance, Golden State Water Company Southwest) in West LA County sub-region.
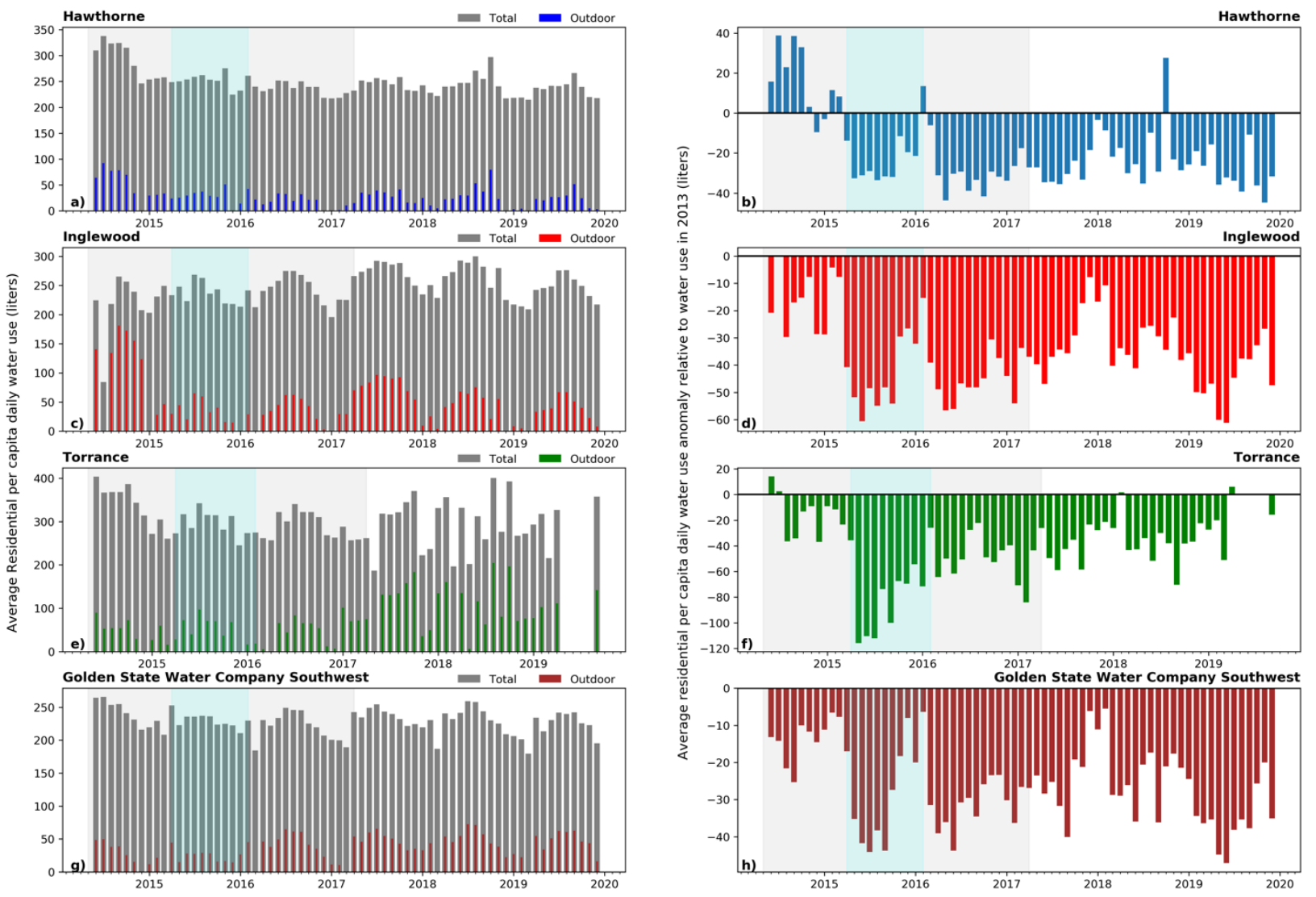
Figure S2: Time series of average ( \pm standard error) residential daily per capita water use, average ( \pm standard error) outdoor residential daily per capita water use (a, c, e, g, i) and percent water use reduction in each month relative to the monthly water deliveries in 2013 (b, d, f, g, j) in urban water suppliers (Long Beach, Newport Beach, Huntington Beach, Seal Beach, Mesa Water District) in Orange County sub-region.
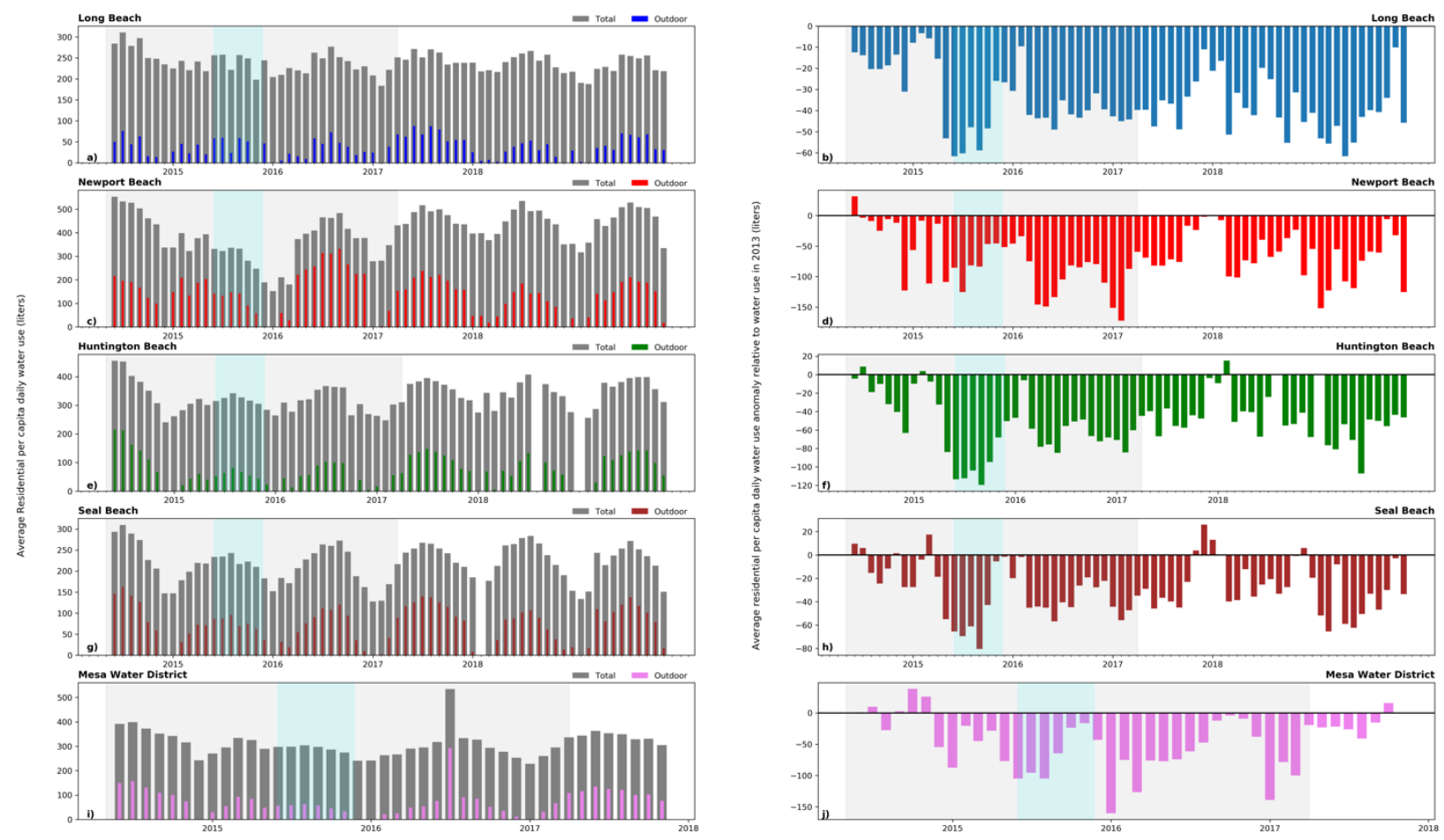
Figure S3: Permutation variable importance from a) West LA, b) Orange County sub-regions showing top 10 environmental predictors.
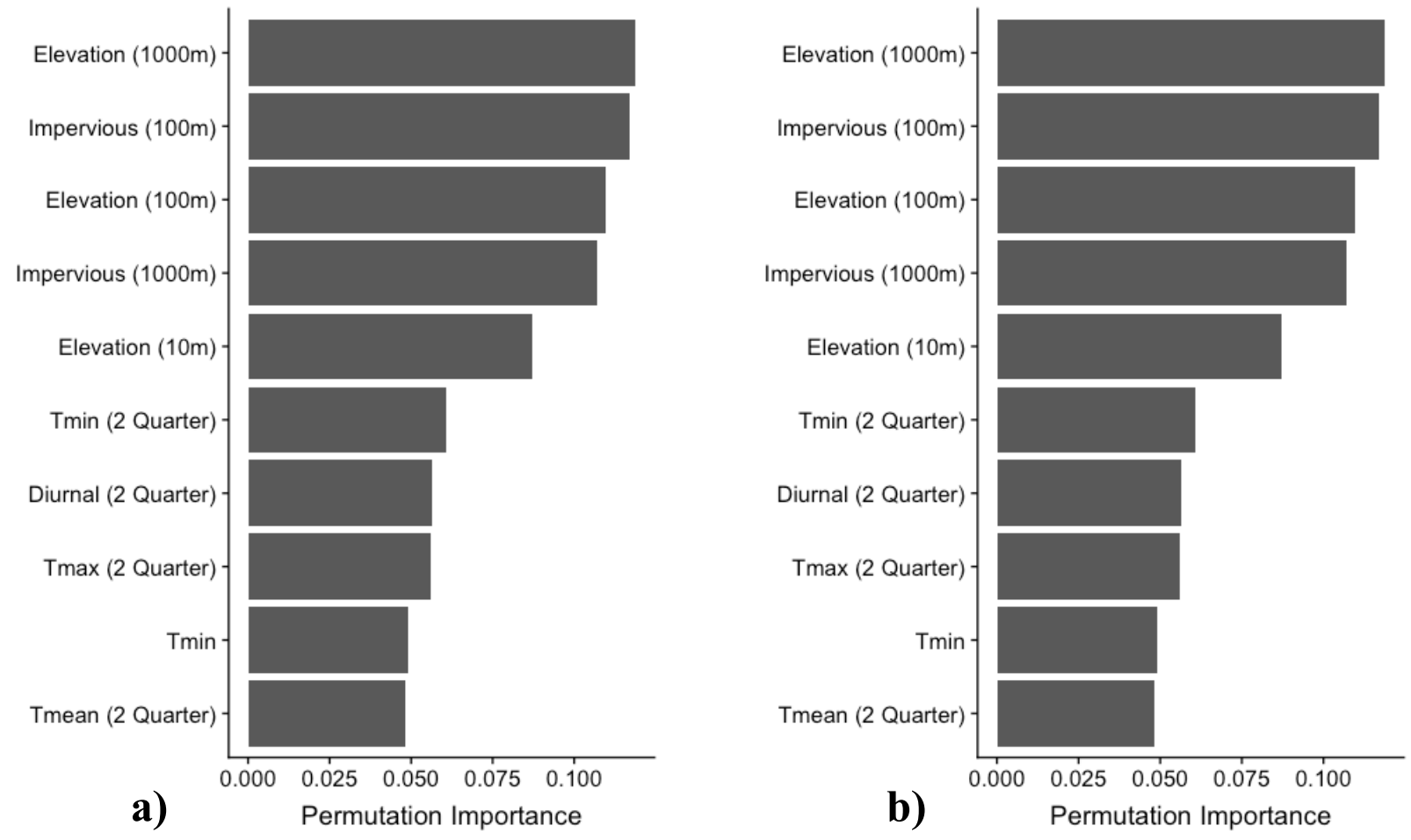